\title{
PROJETO E DESENVOLVIMENTO DE UM MEDIDOR DIGITAL DE ENERGIA ELÉTRICA MONOFÁSICO PARA APLICAÇÕES RESIDENCIAIS
}

\author{
B. R. A. DINIZ ${ }^{*}$ A. A. EVANGELISTA e A. R. ALEXANDRIA \\ Instituto Federal de Educação, Ciência e Tecnologia do Ceará \\ Programa de Pós-graduação em Energias Renováveis \\ bruno.ra.diniz@gmail.com , arturevang@gmail.com, auzuir@ifce.edu.br
}

Submetido 27/09/2016 - Aceito 14/11/2017

DOI: $10.15628 /$ holos.2017.5118

\section{RESUMO}

Acompanhar o consumo de energia elétrica de uma unidade consumidora é um procedimento que ajuda a reduzir os custos energéticos. Ainda que existam, atualmente, meios tecnológicos de se medir e adquirir dados de consumo, os quais abrangem conhecimentos integrados de automação, usa-se técnicas que são tão efetivas quanto as utilizadas em medidores inteligentes. Este artigo descreve e implementa um medidor de energia elétrica digital para uso residencial. Aqui, este equipamento é utilizado como uma importante ferramenta no acompanhamento do consumo e na verificação de eficácia das práticas de eficiência energética adotadas. Para isso, utilizou-se sensores eletrônicos de tensão e corrente e um microcontrolador
\end{abstract}

PIC, responsável por calcular, armazenar e transmitir, por meio de um módulo bluetooth, as medições elétricas realizadas para um software e para um aplicativo, os quais formam a interface do sistema. As medições realizadas pelo protótipo desenvolvido foram verificadas com o uso de instrumentos de medição comerciais e dados de consumo energético de uma residência foram coletados e comparados com 0 fornecido pela concessionária de energia local. Por fim, foi atestado que o sistema realizou o armazenamento dos dados e os enviou ao software corretamente permitindo uma posterior análise através de gráficos, que auxiliam em ações efetivas de eficiência energética.

PALAVRAS-CHAVE: medição de energia elétrica, microcontroladores PIC, sensores eletrônicos e bluetooth.

\section{DESIGN AND DEVELOPMENT OF A SINGLE-PHASE DIGITAL METER FOR RESIDENTIAL APPLICATIONS}

\begin{abstract}
Tracking the energy consumption of a residence is a procedure that helps to reduce the energy costs. Even if there are, currently, technological ways of measuring consumption data, which includes automation concepts, it uses techniques that are as effective as those used in smart meters. This paper describes and implements a single-phase digital meter for residential applications. Here, this device is employed as an important tool for the consumption monitoring and for the verification of the effectiveness of adopted energy efficiency practices. For that, it was used voltage and current electronic sensors, a PIC microcontroller, which is responsible for
\end{abstract}

calculate, store and communicate, by means of a Bluetooth module, the electrical measures to a software and an app, which constitutes the system interface. The measures achieved by the prototype was verified with commercial measuring instruments and energy consumption data of a residence was collected and compared with those supplied by the local power utility. Finally, it was proved that the system performed the data storage and sent it to the software allowing further analysis using graphs, which helps in effective energy efficiency actions. 


\section{INTRODUÇÃO}

Diante do impacto econômico e social causado por seguidos aumentos de contas de energia elétrica e por variadas mudanças nas bandeiras tarifárias, torna-se importante que o consumo de energia seja otimizado através de práticas de eficiência energética e de técnicas que englobam economia de energia (Ochoa, Diniz, Santana, Silva, \& Ochoa, 2015).

Com vistas a atestar a eficácia das práticas de eficiência adotadas, o consumidor precisa acompanhar o consumo de energia elétrica através de recebimento mensal em cobrança da concessionária de energia elétrica. No entanto, esse acompanhamento possui um entrave já que o consumidor somente receberá os resultados atingidos pelas práticas de eficiência uma vez por mês (Santos et al., 2006)

Uma forma de se fazer o acompanhamento de consumo consiste em o consumidor realizar leituras do medidor de energia de sua residência em intervalos menores de tempo (quinzenalmente, semanalmente ou mesmo diariamente) e calcular o consumo desse intervalo. Com isso, elimina-se a desvantagem do método anterior mas acrescentam-se outras tais como a onerosidade, a falta de praticidade do método, a disciplina e o tempo disponível que o consumidor deve possuir. Uma outra alternativa é adquirir um equipamento ou um sistema de monitoramento de energia elétrica. Com isso, pode-se monitorar o consumo individual de cada eletrodoméstico ou de toda a residência. Alguns modelos possuem inclusive integração com tablets, smartphones e até mesmo com a internet, possibilitando para o consumidor um acompanhamento completo e prático de seu consumo energético (Ferreira, 2012).

Em busca de métodos de baixo custo e que sejam eficientes no tocante ao monitoramento de consumo de energia elétrica com vistas à economia de energia, pode-se construir redes de sensores e conectá-los em determinados ambientes em busca de modelar os parâmetros necessários para se calcular o potencial de economia de energia (Batista, Freitas, \& Jota, 2014).

Além de monitoramento de consumo por meio de sensores e modelos matemáticos, costuma-se utilizar redes neurais artificiais que funcionam como mais uma ferramenta importante no controle de sistemas de potência e no monitoramento de parâmetros de estabilidade e qualidade (Hassan, Moghavvemi, Almurib, \& Steinmayer, 2013).

Embora sejam observadas alternativas vantajosas que fazem com que os modelos de medidores utilizados atualmente na maior parte das residências sejam eletrônicos, considera-se que já existem sistemas de medição de consumo de energia residencial chamados de inteligentes e que fazem a integração entre todos os sistemas presentes em uma residência. Além de ser possível permitir a troca de informações e de dados elétricos entre usuários e distribuidoras de energia (Depuru, Wang, \& Devabhaktuni, 2011).

Os medidores inteligentes podem executar diversas funções que são especificadas para que sejam executadas de maneira triunfal tais como medir parâmetros elétricos, armazenar dados que podem ser analisados através de softwares, gravar dados periodicamente e comunicar com usuários remotamente (Aslam, Soban, Akhtar, \& Zaffar, 2015). 
Já é possível implementar uma rede inteligente através do conceito de medidor mais próximo do usuário. Consegue-se incorporar à rede uma plataforma de IOT, integrar rede inteligente com aplicações em automações residenciais e prover acesso seguro para quaisquer usuários do sistema (Spanò, Niccolini, Pascoli, \& Iannacconeluca, 2015).

O medidor digital de energia elétrica residencial proposto nesse trabalho apresenta-se como uma importante ferramenta auxiliar para redução do consumo de energia elétrica, pois uma vez que se tenha acesso a informações sobre o consumo energético de sua residência e sendo essas informações apresentadas de maneira simples e intuitiva, o consumidor passa a acompanhar de perto os resultados de seu racionamento (Ferreira, 2012).

Com o intuito de se adquirir os dados, executou-se o processamento dos sinais em um único microcontrolador, diminuindo a quantidade de componentes e consequentemente o custo além de se desenvolver uma interface do sistema para uso em smartphones e tablets.

O presente artigo é estruturado como segue. A seção 2 é dedicada à explicação das etapas de desenvolvimento do protótipo, desde a concepção do hardware até o desenvolvimento do firmware apresentando detalhes importantes que são cruciais para o correto funcionamento de ambos. As interfaces do software e do aplicativo também são explicadas nesta seção. Na seção 3 submete-se o medidor de energia desenvolvido a experimentos, usando como referência equipamentos comerciais e realiza-se também o teste de funcionalidade, onde o medidor é posto em operação por vários dias e seus dados gerados são coletados e analisados. Por fim, na seção 4, sugere-se modificações e aperfeiçoamentos tanto no hardware, quanto no software assim como também no aplicativo visando melhorias na aparência e aumento de funcionalidades. Estas normas têm como objetivo dar uma orientação geral aos autores dos artigos no momento em que forem redigir e, principalmente, quando forem organizar e digitar seus artigos científicos.

\section{MATERIAIS E MÉTODOS}

\subsection{Diagrama de blocos}

Na Figura 1, é mostrado o diagrama de blocos simplificado do hardware do medidor de energia e a seguir, uma explicação superficial de cada bloco representado.

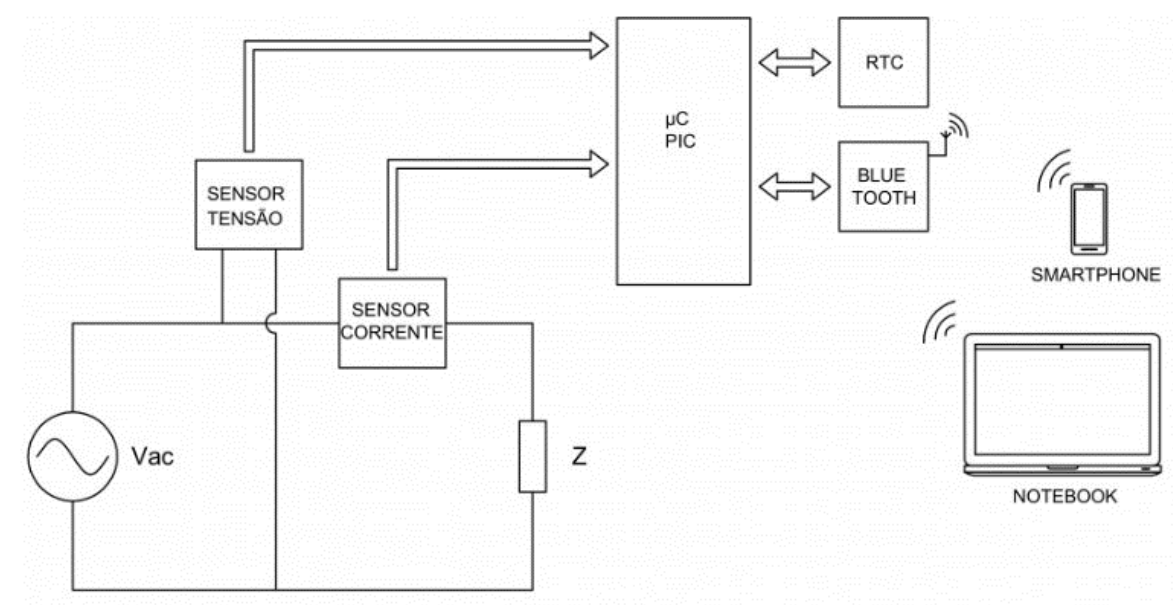

Figura 1: Diagrama de blocos do medidor de energia. 
O circuito elétrico monitorado é representado pela fonte de tensão alternada "Vac" e pela carga Z com os sensores de tensão e corrente representados respectivamente pelos blocos SENSOR TENSÃO e SENSOR CORRENTE. O microcontrolador é mostrado no bloco $\mu C$ PIC o qual é responsável pela aquisição dos sinais dos sensores e processamento daqueles, obtendo como resultado os valores de tensão, corrente e potência do circuito monitorado.

O microcontrolador, em conjunto com o relógio em tempo real representado no bloco RTC, calcula a energia consumida a cada hora e armazena em memória interna não volátil. $O$ módulo bluetooth possibilita a comunicação do microcontrolador com um PC ou com um smartphone, onde se encontra a interface do sistema e podem ser visualizadas todas as informações calculadas ou armazenadas.

\subsection{Sensores}

Para o cálculo da potência e energia consumida em um circuito baseado em corrente alternada são necessárias três informações: tensão, corrente e tempo. Os circuitos descritos nos tópicos seguintes realizam a aquisição das duas primeiras informações e as tornam compatíveis para processamento por um microcontrolador.

\subsubsection{Circuito sensor de corrente}

O circuito desenvolvido é mostrado na Figura 2, baseado no componente ACS712-30 que é um sensor de corrente por efeito Hall capaz de medir correntes de até 30 A. Fornece no pino VIOUT um valor de tensão proporcional à corrente aplicada através dos terminais IP+ e IP-, sendo sua sensibilidade, ou seja, a relação entre a tensão de saída e a corrente de entrada, de tipicamente igual a $66 \mathrm{mV} / \mathrm{A}$ e seu erro máximo de aproximadamente 1,5\% à temperatura de $25^{\circ} \mathrm{C}$. Na ausência de qualquer corrente, mantém uma tensão de offset de $\mathrm{Vcc} / 2$ Volts, sendo $\mathrm{Vcc}$ a tensão de alimentação desse componente. Como $\mathrm{Vcc}$ é igual a $5 \mathrm{~V}$, a tensão de offset é $2,5 \mathrm{~V}$ (Microsystems, 2017).

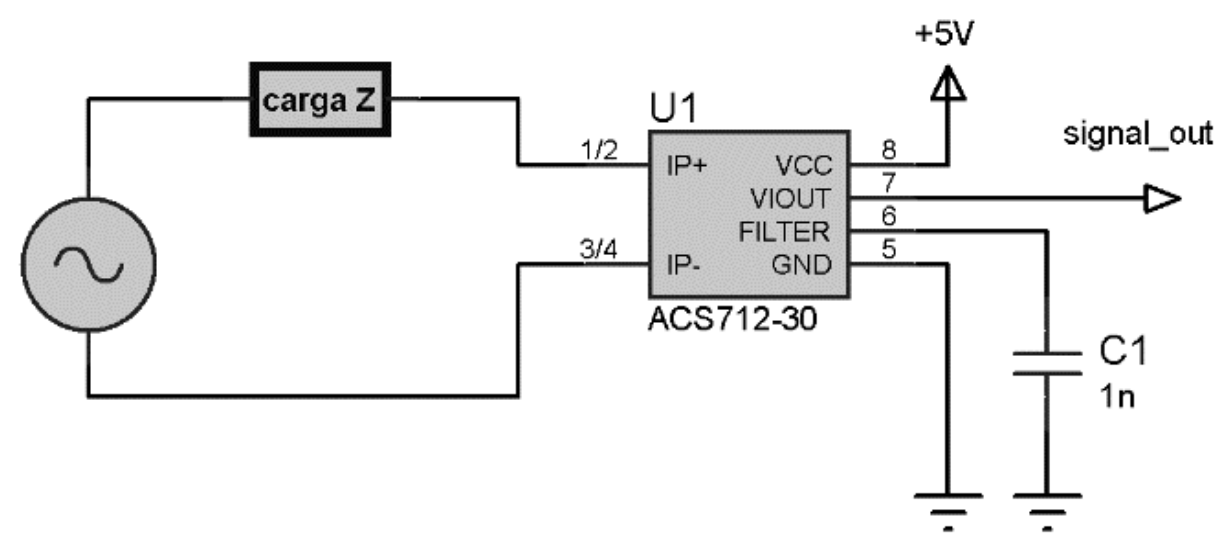

Figura 2: Esquema elétrico do circuito sensor de corrente Fonte: (Microsystems, 2017).

\subsubsection{Circuito sensor de tensão}

O circuito sensor de tensão, projetado para operar com tensões de entrada de até $250 \mathrm{~V}$, é mostrado na Figura 3 e tem como principal componente um transformador, utilizado para 
baixar a tensão de entrada de $220 \mathrm{~V}$ no enrolamento primário para $3 \mathrm{~V}$ no secundário, com capacidade de corrente igual a $200 \mathrm{~mA}$. O varistor TVR10391 é projetado para operar a uma tensão máxima de $250 \mathrm{~V}$. Para tensões de pico acima de $650 \mathrm{~V}$, o componente entra em operação desviando a corrente do transformador e protegendo todo o circuito.

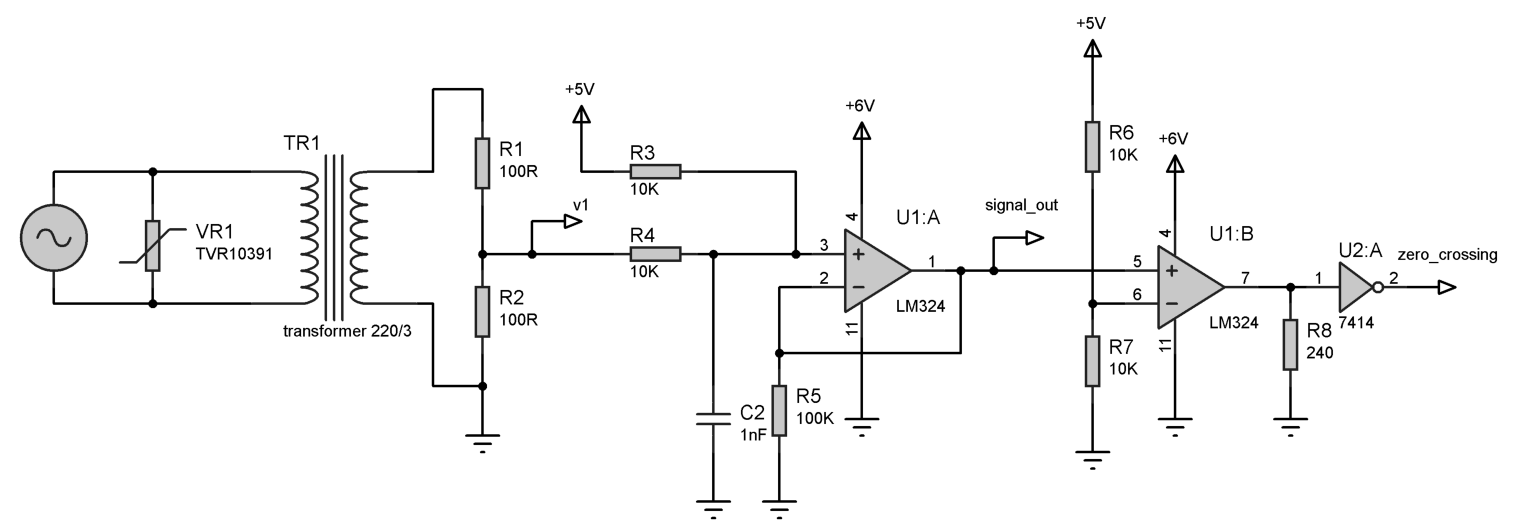

Figura 3: Esquema elétrico do circuito sensor de tensão.

Através dos resistores R1 e R2, a tensão de saída do transformador é dividida por 2 e representada na Figura 3 como v1. É nessa parte do circuito que é adicionada uma referência através da conexão do GND à R2. O amplificador operacional LM324 nomeado como U1:A está na configuração somador não inversor com ganho unitário e tem a função de retirar a parte negativa do sinal v1 através da soma de um valor de tensão fixo, um offset, que assim como no circuito sensor de corrente, tem valor de 2,5 V. Para correto funcionamento desse circuito, os resistores R3 e R4 devem ter exatamente o mesmo valor, então foram usados resistores com precisão de $0,1 \%$.

\subsection{Microcontrolador}

O microcontrolador utilizado no medidor de energia foi o PIC18F26K80. Esse microcontrolador de 8 bits da Microchip possui três características que são determinantes para proporcionar uma boa performance ao sistema como um conversor analógico digital (ADC) com resolução de 12 bits, o que incrementa a resolução da conversão, frequência de operação de até $64 \mathrm{MHz}$, executando 16 milhões de instruções por segundo (MIPS) e Multiplicador 8 bits em hardware em apenas um ciclo de máquina (Microchip, 2011).

\subsection{Firmware}

O firmware embarcado no microcontrolador tem a função de coletar os dados dos sensores, fazer o tratamento dos mesmos e obter os valores das grandezas elétricas do circuito monitorado pelo medidor de energia, além de prover a comunicação com software ou aplicativo através de um módulo bluetooth e com o RTC (Real Time Clock) através do protocolo $\mathrm{I}^{2} \mathrm{C}$. $\mathrm{O}$ firmware foi todo desenvolvido em linguagem $C$ utilizando-se o compilador XC8 e o ambiente de desenvolvimento MPLABX. Na Figura 4, pode-se ver o fluxograma simplificado de sua rotina principal. 


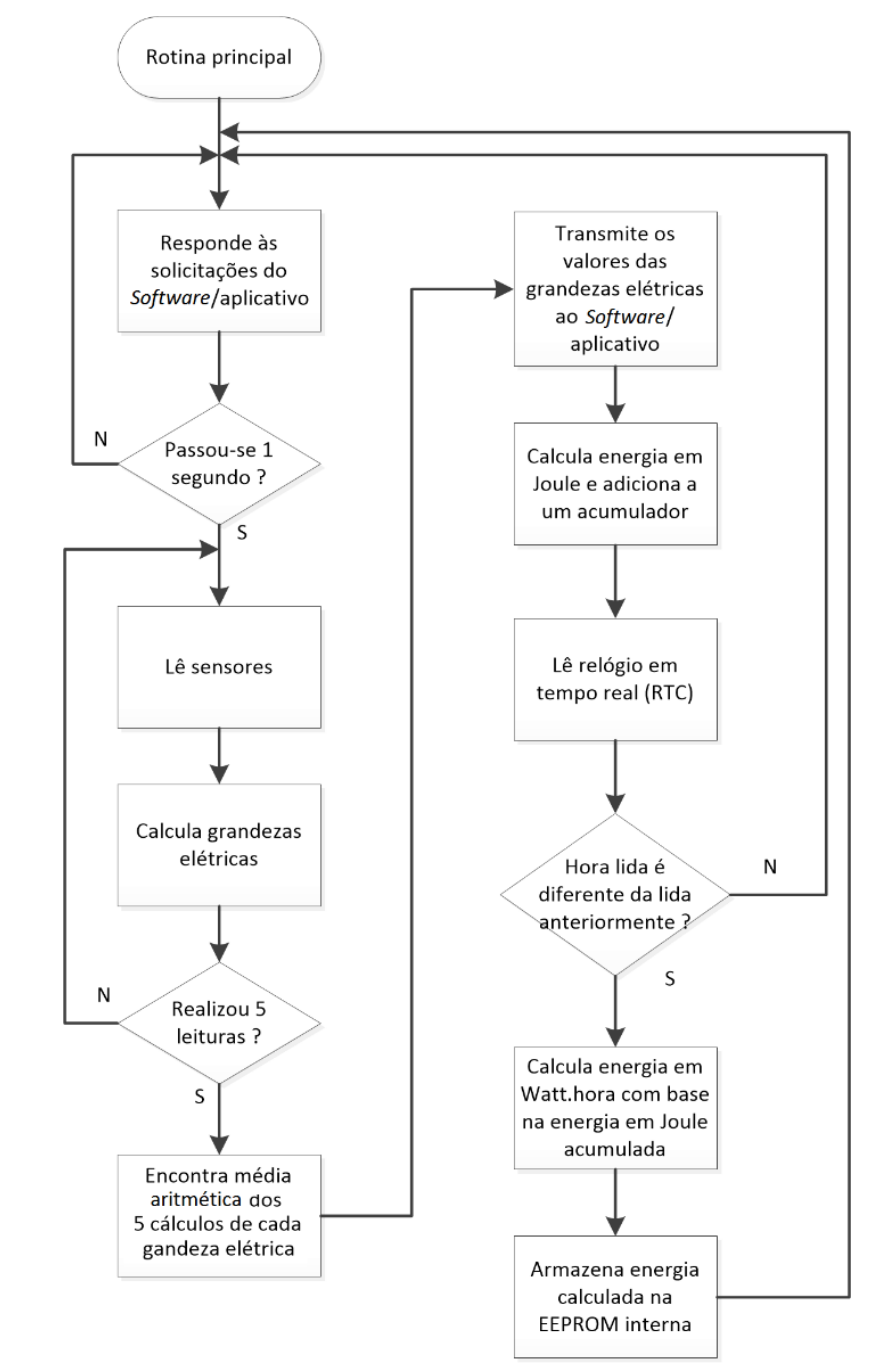

Figura 4: Fluxograma da rotina principal do firmware.

\subsection{Interface}

O medidor de energia não possui em seu hardware uma interface que permita ao usuário a visualização das medições realizadas, para isso foram desenvolvidos um software, concebido em plataforma JAVA, e um aplicativo compatível com a plataforma Android versão 4.0. A função deles é, além de exibir as medições realizadas, permitir ao usuário configurar certos parâmetros do medidor.

Observa-se que na Figura 5a há um campo para permitir a conexão com o medidor de energia, que é feita através de uma porta serial previamente criada pelo sistema operacional após pareamento com o bluetooth do medidor. No campo "porta" digita-se o nome da porta serial e clica-se em "conectar"; após isso, tensão, corrente, potência ativa, potência aparente e fator de potência passam a ser exibidos com uma taxa de atualização de $1 \mathrm{~Hz}$. É possível interromper o recebimento dessas informações ao clicar no botão "Desabilitar" e voltar a recebêlas, clicando novamente no mesmo botão, que agora terá o nome "Habilitar". É possível ver na Figura 5b, a aba de configuração. A partir dela, é possível sincronizar o horário do RTC de acordo com o relógio do PC através do botão "Enviar Data e Hora" e também visualizar o horário atual do RTC através do botão "Solicita Data e Hora". A calibração do medidor também é realizada nessa aba e o procedimento para tal é descrito a seguir. 


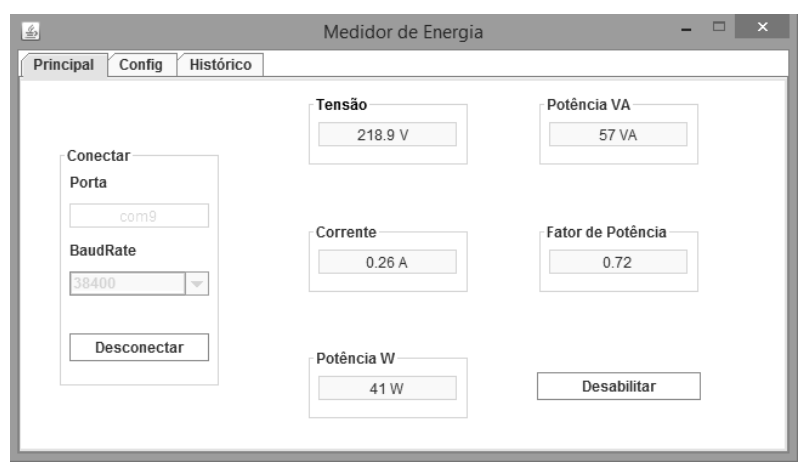

a)

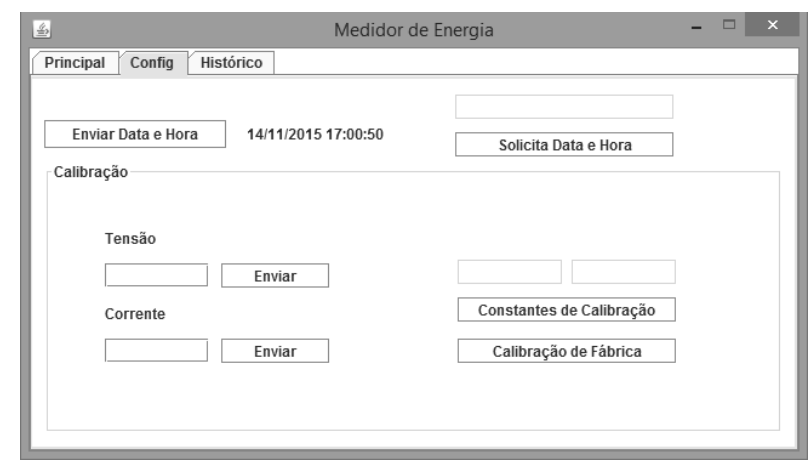

b)

Figura 5: Software desenvolvido - a) Aba principal; b) Tela principal

Inicialmente, deve-se ter conectados à carga, juntamente com o medidor de energia, um voltímetro e um amperímetro. A carga deve ser alimentada, e então as medidas realizadas pelo voltímetro e amperímetro devem ser digitadas nos campos "Tensão" e "Corrente" respectivamente. Após clicar em "enviar" o medidor já se encontra calibrado. Se desejável, podem ser solicitados os valores das resoluções de tensão e corrente através do botão "Constantes de Calibração". É possível voltar à calibração padrão ao clicar em "Calibração de Fábrica".

A aba Histórico é mostrada na Figura 6. Esta aba é responsável por exibir o histórico das medições de energia realizadas pelo microcontrolador. Ao clicar em "solicitar", todos os pacotes de energia armazenados na EEPROM são enviados ao software e exibidos na tela.

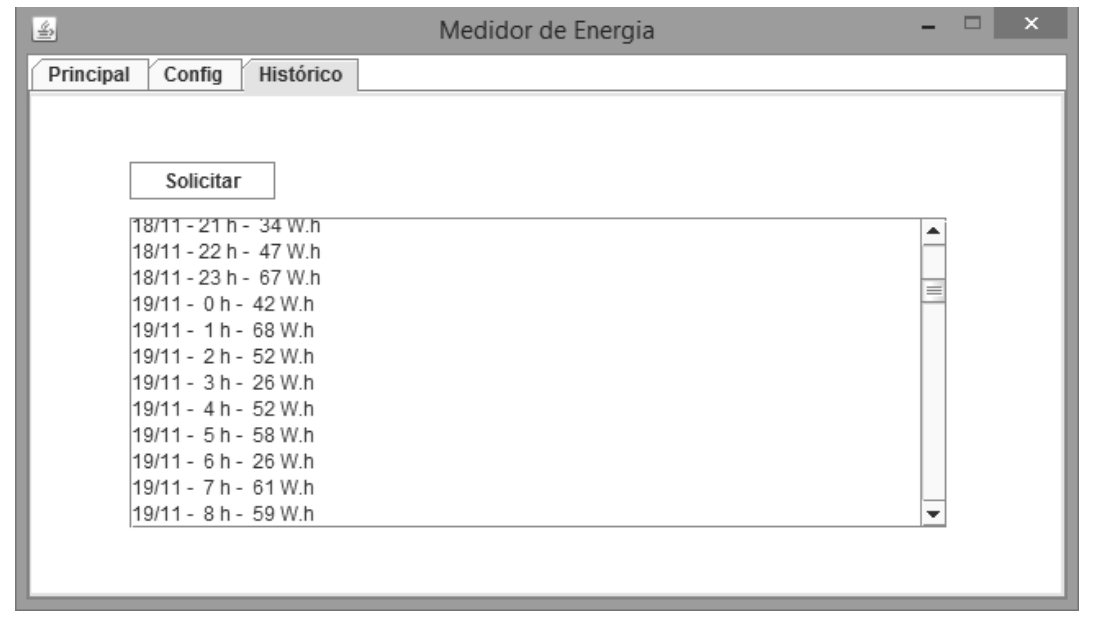

Figura 6: Aba de histórico de medições de energia do software desenvolvido.

Assim que aberto, caso o bluetooth do dispositivo não esteja ativado, o aplicativo solicita permissão para fazer isso. Com a permissão concedida, a tela principal é exibida e faz-se necessária a conexão com o medidor de energia que deve ser realizada através de um menu, que é exibido quando se toca em um ícone com três pontos no canto superior direito da tela. Após conexão, os valores enviados pelo medidor de energia são continuamente exibidos com um intervalo de 1 segundo na tela principal do aplicativo conforme visto na Figura 7. Na mesma tela, é possível realizar a calibração do medidor, enviando os valores de tensão e corrente medidos 
com um voltímetro e amperímetro, respectivamente, nos campos situados no inferior da tela e em seguida tocando no botão correspondente ao que se deseja calibrar.

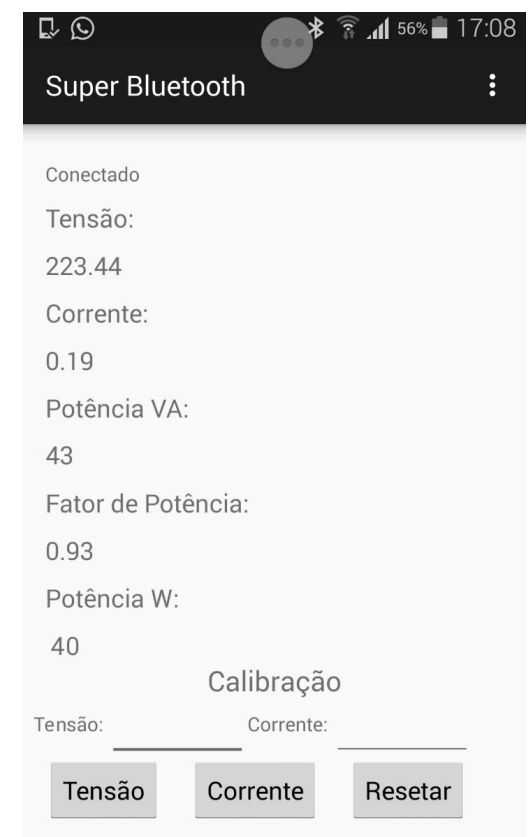

Figura 7: Tela principal do aplicativo com os valores exibidos depois da conexão do medidor.

\section{RESULTADOS}

\subsection{Ensaio de Tensão}

Para verificação da tensão, foi montado o circuito cujo diagrama é mostrado na Figura 8, composto por uma fonte de tensão alternada ajustável, um voltímetro, uma carga e o medidor de energia.

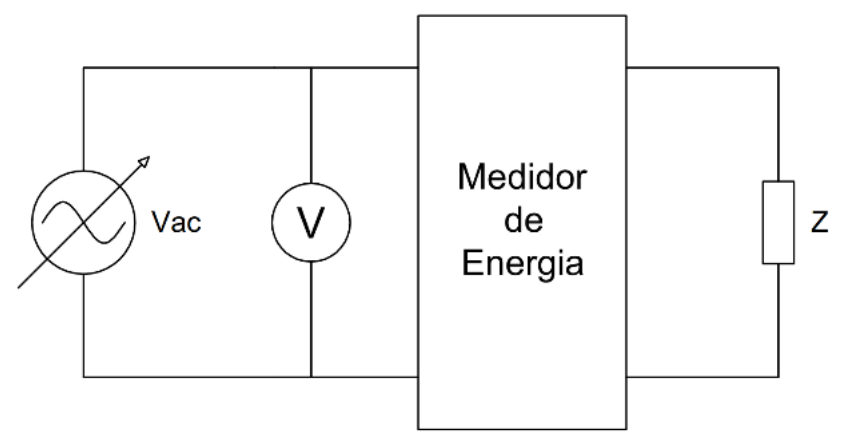

Figura 8: Circuito para verificação da tensão.

Utiliza-se o multímetro modelo ET2082C do fabricante Minipa, que, apesar de não ser TRUE RMS, pode ser utilizado nesse teste, visto que as tensões medidas são senoidais.

Inicialmente, é necessário calibrar o medidor de energia tendo como referência o multímetro na escala 200 Vac. A calibração foi realizada à tensão de 137,4 V. Após esse 
procedimento, foram realizadas diversas medições de diferentes tensões, os resultados são mostrados na Tabela 1.

A resolução do multímetro ET2082C na escala de $200 \mathrm{~V}$ é de $100 \mathrm{mV}$.

Tabela 1: Comparação entre medições de tensão realizadas pelo multímetro e pelo medidor de energia até $200 \mathrm{~V}$.

\begin{tabular}{c|c}
\hline Medição do multímetro (V) & Medição do medidor de energia (V) \\
\hline 20,3 & 20,7 \\
\hline 40,4 & 40,8 \\
\hline 60,2 & 60,4 \\
\hline 80,1 & 80,3 \\
\hline 100,2 & 100,5 \\
\hline 120,0 & 120,4 \\
\hline 140,2 & 140,5 \\
\hline 160,5 & 160,4 \\
\hline 180,6 & 180,4 \\
\hline 199,4 & 199,1
\end{tabular}

Para testes com tensões superiores a $200 \mathrm{~V}$, utilizou-se a escala 750 Vac do multímetro. Sendo assim, uma nova calibração foi realizada no medidor de energia dessa vez utilizando $237 \mathrm{~V}$ como fator de escala. A Tabela 2 exibe os resultados das medições acima de $200 \mathrm{~V}$. A resolução do multímetro ET2082C na escala de $750 \mathrm{~V}$ é de $1 \mathrm{~V}$.

Tabela 2: Comparação entre medições de tensão realizadas pelo multímetro e pelo medidor de energia acima de $200 \mathrm{~V}$.

\begin{tabular}{c|c}
\hline Medição do multímetro (V) & Medição do medidor de energia (V) \\
\hline 200 & 200,8 \\
\hline 210 & 210,6 \\
\hline 220 & 220,7 \\
\hline 230 & 230,9
\end{tabular}

\subsection{Ensaio de corrente}

O circuito representado pelo diagrama mostrado na Figura 9, composto por uma fonte de tensão fixa, um amperímetro, várias cargas (porém apenas uma conectada por vez) e o medidor de energia foi utilizado para medir a corrente.

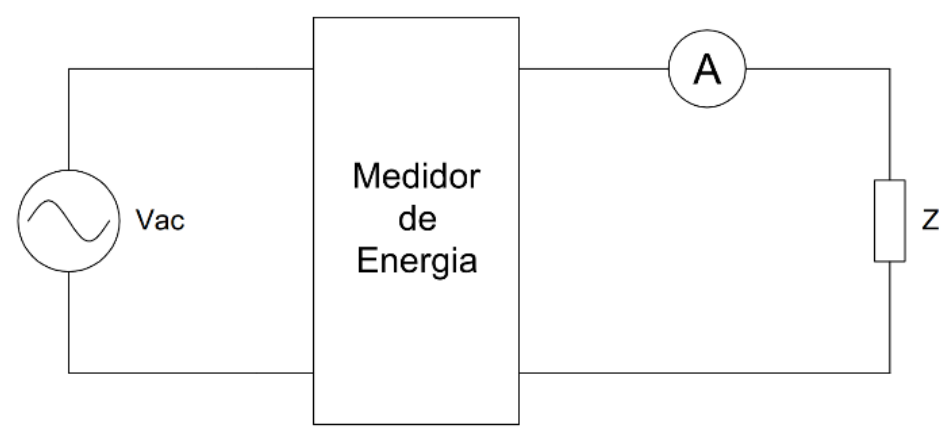

Figura 9: Circuito para verificação da corrente. 
Utilizou-se o multímetro modelo HM-2090 do fabricante Hikari, que possui resolução igual a $10 \mathrm{~mA}$, na escala de $10 \mathrm{~A}$. A calibração foi realizada com corrente igual a 4,32 A para uma carga resistiva. A Tabela 3 apresenta medidas de correntes elétricas de alguns equipamentos de uso comum em uma residência.

Tabela 3: Comparação entre medições de corrente realizadas pelo multímetro e pelo medidor de energia.

\begin{tabular}{c|c|c}
\hline Descrição da Carga & Medição do multímetro (A) & Medição do medidor de energia (A) \\
\hline Sanduicheira & 3,01 & 3,02 \\
\hline TV tubo 14" & 0,36 & 0,35 \\
\hline Liquidificador & 0,68 & 0,67 \\
\hline Computador de mesa & 0,65 & 0,65 \\
\hline Notebook & 0,31 & 0,30 \\
\hline Ventilador & 0,18 & 0,20 \\
\hline TV LED 32" & 0,19 & 0,19 \\
\hline Batedeira & 0,60 & 0,61 \\
\hline Furadeira & 1,00 & 1,01 \\
\hline Forno de Micro-ondas & 5,22 & 5,22 \\
\hline Geladeira & 0,65 & 0,67 \\
\hline Ferro Elétrico & 4,56 & 4,55 \\
\hline Lâmpada LED 12 W & 0,08 & 0,09 \\
\hline Lâmpada Fluorescente 20 W & 0,15 & 0,16
\end{tabular}

\subsection{Ensaio de potência}

O circuito visto na Figura 10 composto por uma fonte de tensão alternada, um wattímetro, o medidor de energia e diferentes cargas conectadas uma por vez foi utilizado para a verificação das potências.

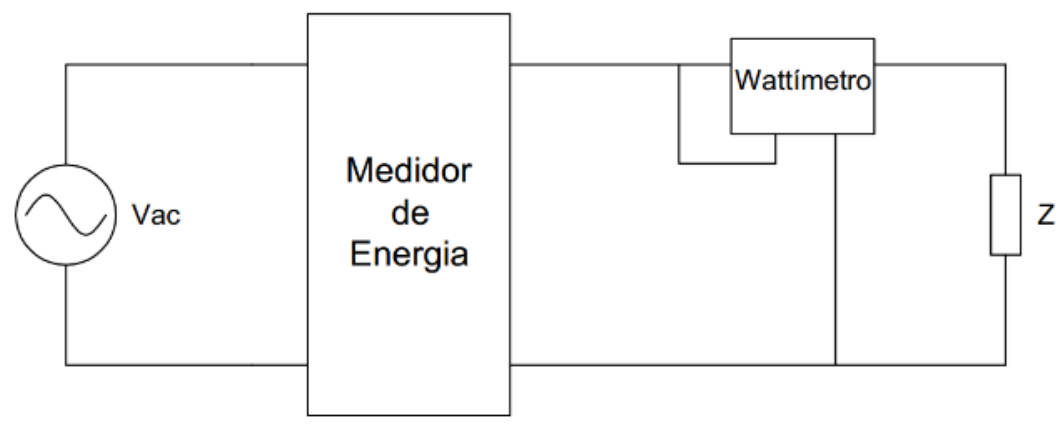

Figura 10: Circuito para verificação das potências.

Utilizou-se o wattímetro TRUE-RMS modelo ET-4080 do fabricante Minipa, capaz de medir tensão, corrente, potências ativa, reativa e aparente, além de medir também fator de potência e frequência. Esse instrumento, no entanto, apesar de ser capaz de medir correntes de até $1000 \mathrm{~A}$, possui a limitação de corrente mínima de $1 \mathrm{~A}$, ou seja, só é capaz de exibir valores de corrente e potências de cargas que consumam pelo menos $1 \mathrm{~A}$. As resoluções das potências para a faixa utilizada durante a verificação são de 10 unidades (10 W, 10 VA e 10 VAr). Assim como na verificação de tensão e corrente, o medidor de energia foi calibrado tendo como referência o instrumento, nesse caso o wattímetro. Devido à limitação de corrente mínima medida pelo ET- 
4080, a quantidade de cargas disponíveis para teste foi limitada. A Tabela 4 mostra os resultados obtidos para o medidor e para o wattímetro.

Tabela 4: Comparação entre medições de corrente realizadas pelo wattímetro e pelo medidor de energia

\begin{tabular}{c|c|c|c}
\hline Descrição da carga & $\begin{array}{c}\text { Potência ativa } \\
(\mathrm{kW})\end{array}$ & $\begin{array}{c}\text { Medição do Wattímetro } \\
(\mathrm{kW})\end{array}$ & $\begin{array}{c}\text { Medidor de energia } \\
(\mathrm{kW})\end{array}$ \\
\hline Prensa térmica & 1,32 & 1,312 & 1,317 \\
\hline $\begin{array}{c}\text { Lâmpadas } \\
\text { Fluorescentes }\end{array}$ & 0,25 & 0,255 & 0,255 \\
\hline
\end{tabular}

\subsection{Ensaio de energia}

Por último, verificou-se, no medidor de energia, a energia (kWh). Para isso, o medidor foi instalado em um apartamento, de modo que se pudesse realizar a medição de toda a energia consumida. Posteriormente, os dados adquiridos foram posteriormente comparados com os medidos pelo medidor instalado na parte externa da residência que pertence à Companhia Energética do Ceará (COELCE). O esquema de instalação é mostrado na Figura 11 e o medidor de energia é visto na Figura 12.

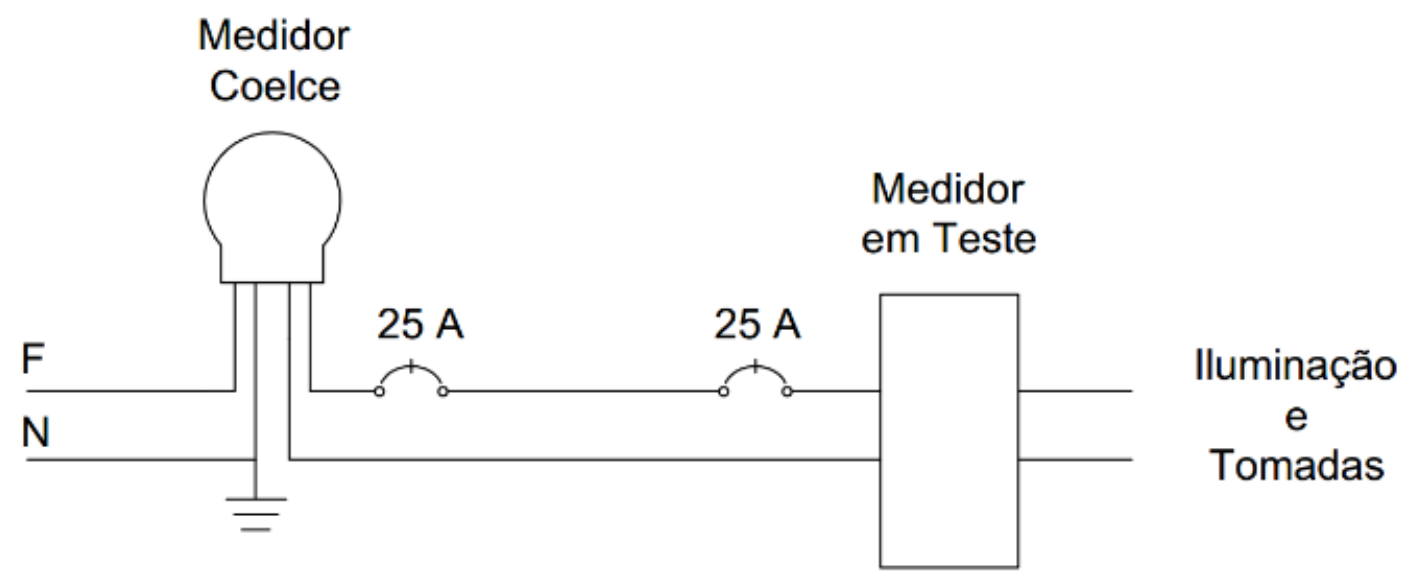

Figura 11: Circuito para verificação da energia. 


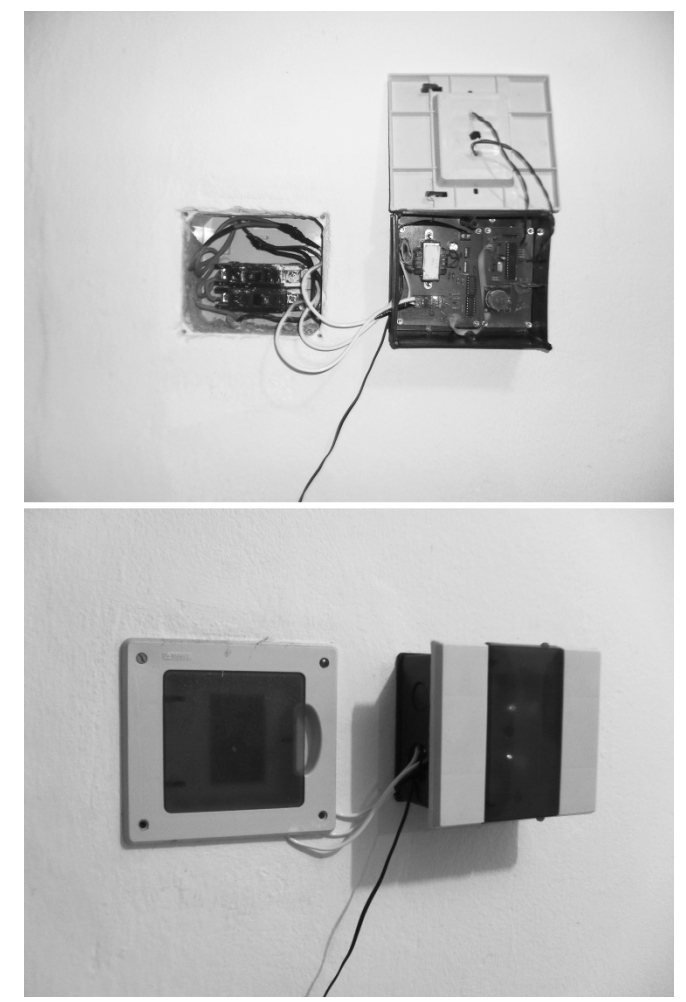

Figura 12: Medidor de energia instalado.

O medidor apresentado na Figura 12 foi deixado em funcionamento por 4 semanas e durante esse período foram realizadas 4 leituras do medidor em teste e do medidor da COELCE para fins de comparação. Todas as leituras realizadas são apresentadas na Tabela 5.

Tabela 5: Comparação entre medições de energia realizadas pelo medidor de energia em teste e o medidor de energia da COELCE.

\begin{tabular}{c|c|c|c}
\hline Data Inicial & Data Final & Medidor COELCE $(\mathrm{kWh})$ & Medidor em teste $(\mathrm{kWh})$ \\
\hline $28 / 12 / 2015$ & $03 / 01 / 2016$ & 37,9 & 36,36 \\
\hline $03 / 01 / 2016$ & $09 / 01 / 2016$ & 37 & 35,28 \\
\hline $09 / 01 / 2016$ & $17 / 01 / 2016$ & 46 & 43,92 \\
\hline $17 / 01 / 2016$ & $23 / 01 / 2016$ & 35 & 33,32 \\
\hline
\end{tabular}

\section{CONCLUSÃO}

Foi desenvolvido e apresentado neste artigo um equipamento de medição de energia para uso residencial de baixo custo e interface amigável com integração a computadores, tablets e smartphones. A solução apresentada envolveu o uso de sensores e outros dispositivos eletrônicos que, aliados a uma programação eficiente e a uma interface externa, permitiram a redução de custos, aumento de funcionalidades e desempenho satisfatório.

No ensaio de tensão, alcançou-se, em determinadas medidas, erros com limites máximos iguais a $2 \%$. Considera-se que os erros encontrados foram baixos principalmente quando estes são comparados, para fins de medição de faturamento, com os valores encontrados em classes de exatidão de transformadores de potencial, que devem possuir erros menores para que não haja medidas impraticáveis. Para a medição de corrente, os erros encontrados foram ainda menores que aqueles do ensaio de tensão, comprovando a boa exatidão do medidor digital. 
As mesmas considerações são feitas para as medições de potência e de energia, embora os resultados das medições de energia tenham sido prejudicados pela ausência de calibração entre o medidor desenvolvido e o da distribuidora de energia. Apesar disso, atingiu-se erros iguais a $5 \%$, limite aceitável quando se considera uma variação de $5 \%$ entre as tensões permitidas por norma.

Quanto à funcionalidade do sistema, foi atestado que ele funcionou dentro do esperado, pois realizou o armazenamento dos dados e os enviou ao software corretamente permitindo uma posterior análise através de gráficos.

Deve-se considerar que como o projeto não se destina a medir energia para fins tarifários, ou seja, o medidor de energia desenvolvido apenas serve como uma ferramenta auxiliar no acompanhamento do consumo energético, atribui-se a sua importância principalmente para o usuário residencial que desejar automatizar o monitoramento do consumo e reduzir o custo da fatura de energia bem como para os consumidores industriais que buscam otimizar custos com a energia elétrica.

Como trabalhos futuros, sugere-se acrescentar ao circuito uma memória não volátil que permita o armazenamento de pelo menos um mês de histórico de consumo energético além de utilizar um microcontrolador com um conversor AD de maior resolução, de maneira que levem os erros de medição a valores próximos de zero também, pode-se ainda trabalhar na miniaturização do circuito utilizando a tecnologia SMT (Surface-Mount Technology), além de utilizar acopladores ópticos como sensores de correntes. Por fim, aperfeiçoar o software e o aplicativo através do melhoramento de sua interface, acréscimo de gráficos e até mesmo implementação de algoritmos que identifiquem o padrão de consumo da residência e informe ao usuário qualquer alteração deste.

\section{REFERÊNCIAS}

Aslam, W., Soban, M., Akhtar, F., \& Zaffar, N. A. (2015). Smart meters for industrial energy conservation and efficiency optimization in Pakistan: Scope, technology and applications. Renewable and Sustainable Energy Reviews, 44, 933-943. http://doi.org/10.1016/j.rser.2015.01.004

Batista, A. P., Freitas, M. E. A., \& Jota, F. G. (2014). Evaluation and improvement of the energy performance of a building's equipment and subsystems through continuous monitoring. Energy and Buildings, 75, 368-381. http://doi.org/10.1016/j.enbuild.2014.02.029

Depuru, S. S. S. R., Wang, L., \& Devabhaktuni, V. (2011). Smart meters for power grid: Challenges, issues, advantages and status. Renewable and Sustainable Energy Reviews, 15(6), 27362742. http://doi.org/10.1016/j.rser.2011.02.039

Ferreira, J. B. (2012). Análise de Formas de Medição de Consumo de Energia Elétrica no Setor Residencial.

Hassan, L. H., Moghavvemi, M., Almurib, H. a. F., \& Steinmayer, O. (2013). Current state of neural networks applications in power system monitoring and control. International Journal of Electrical Power \& Energy Systems, 51, 134-144. http://doi.org/10.1016/j.ijepes.2013.03.007

Microchip. (2011). PIC18F66K80 Family Data Sheet.

Allegro Microsystems. (2017). Acs712.

Ochoa, A. A., Diniz, H., Santana, W., Silva, P., \& Ochoa, L. (2015). Aplicação De Uma Fonte 
Alternativa De Energia Termelétrica a Gás Natural Visando Reduzir O Custo Com Energia Elétrica Em Um Edifício Comercial. Holos, 1(0), 72. http://doi.org/10.15628/holos.2015.2362

Santos, A. H. M., Simões, A. A., Martins, A. R. S., Viana, A. N. C., Ferreira, C., Bortoni, E. da C., ... Silva, V. F. da. (2006). Conservação de Energia: Eficiência Energética de Equipamentos e Instalações ( $3^{\mathrm{a}}$ Edição). Itajubá: Eletrobrás / PROCEL EDUCAÇÃO.

Spanò, E., Niccolini, L., Pascoli, S. Di, \& lannacconeluca, G. (2015). Last-meter smart grid embedded in an internet-of-things platform. IEEE Transactions on Smart Grid, 6(1), 468-476. http://doi.org/10.1109/TSG.2014.2342796 